\title{
Efficacy and safety of Nivolumab in non-small cell lung cancer patients in Tel-Aviv tertiary medical center: Facing the reality
}

\author{
SIVAN SHAMAI and OFER MERIMSKY \\ Unit of Bone and Soft Tissue Oncology, Division of Oncology, Tel-Aviv Sourasky Medical Center, \\ Affiliated with Sackler School of Medicine, Tel-Aviv University, Tel-Aviv 64239, Israel \\ Received January 10, 2018; Accepted July 2, 2018
}

DOI: $10.3892 / \mathrm{mco} .2018 .1693$

\begin{abstract}
Nivolumab is a human IgG4 programmed death (PD)-1 immune checkpoint inhibitor antibody, which is approved in Israel for the treatment of patients with advanced non-small cell lung cancer (NSCLC). It is also administered to individuals with disease progression during or after platinum-based chemotherapy, without a need to determine the level of PD-L1 expression in the tumor. The present study aimed to evaluate the survival and efficacy of Nivolumab treatment. A retrospective analysis was performed at a thoracic oncology service in a tertiary referral center (Tel-Aviv Sourasky Medical Center), on patients with NSCLC (squamous and non-squamous). All patients were treated with Nivolumab $3 \mathrm{mg} / \mathrm{kg}$, administered intravenously every 2 weeks as part of a compassionate use program. The survival data was analyzed after 22 months. The overall survival (OS) was 34.9\%, while the progression free survival (PFS) was $19.3 \%$. The median PFS from the first dose of Nivolumab to treatment discontinuation was 4 months. A response assessment was performed in the 62 patients who received at least four cycles of Nivolumab, out of the 77 patient cohort. There was a complete response in 1 patient, a partial response in 11 patients, stable disease in 25 patients and progressive disease in 25 patients. The observed response rate of Nivolumab as a service treatment in unselected patients with unknown PD-L1 status NSCLC was $19 \%$. The disease control rate was $60 \%$. In the present study Nivolumab was given to a cohort of patients representing those seen in daily clinical practice, as opposed to a clinical trial setting. Survival and efficacy results strongly support the continued use of Nivolumab as a treatment for NSCLC.
\end{abstract}

Correspondence to: Professor Ofer Merimsky, Unit of Bone and Soft Tissue Oncology, Division of Oncology, Tel-Aviv Sourasky Medical Center, Affiliated with Sackler School of Medicine, Tel-Aviv University, 6 Weitsman Street, Tel-Aviv 64239, Israel E-mail: merimsky@zahav.net.il

Key words: non-small cell lung cancer, immunotherapy, nivolumab, real-life data

\section{Introduction}

Prior to Programmed Death 1 (PD1) and Programmed Death Ligand 1 (PDL1) immune checkpoint inhibitors development, Non Small Cell Lung Cancer (NSCLC) stage IV patients faced a grim prognosis following resistance to platinum based chemotherapy. Standard second line treatment options included Docetaxel (1), Pemetrexed (2) or Epidermal growth factor tyrosine kinase inhibitors $(3,4)$ and median overall survival did not exceed 8 months, at the expense of significant toxicity.

PD1 and PDL1 inhibitors changed all of that in several pivotal trials. Nivolumab, a human IgG4 programmed death 1 immune checkpoint inhibitor antibody, is now approved in Israel as well as other parts of the world, for the treatment of patients with advanced NSCLC and disease progression despite platinum-based chemotherapy, without a need to determine the level of PD-L1 expression in the tumor. The CheckMate 057 trial enrolled 582 patients who were randomized to receive Nivolumab or Docetaxel. Nivolumab demonstrated improved overall survival (OS) compared with Docetaxel with a hazard ratio $(\mathrm{HR})$ of $0.73(\mathrm{P}=.0015)$, and a median OS of 12.2 months in patients treated with Nivolumab compared with 9.4 months in patients treated with Docetaxel $(5,6)$.

In this article, we retrospectively analyzed the results of treating NSCLC patients with Nivolumab. This real lifedata represents, the outcome of a compassionate use program, utilizing broad inclusion criteria.

\section{Patients and methods}

Patients. A retrospective analysis was carried out in a thoracic oncology service in a tertiary referral center (Tel-Aviv Sourasky Medical Center), on patients with NSCLC, both squamous and non-squamous subtypes. All patients were treated with Nivolumab as part of a generous compassionate use program supported by BMS.

Inclusion criteria were patients with documented NSCLC, and a performance status of Eastern Cooperative Oncology Group (ECOG) 0-4. The cohort included both positive and negative pdl1 tumors, as well as any EGFR/ALK/ROS1 mutation status. Patients were previously treated with platinum based combination chemotherapy with documented clinical 
and radiological disease progression. They had adequate organ functions as reflected by blood count and biochemical serum analysis (creatinine, ALT, AST). Exclusion criteria were active autoimmune diseases, treatment with systemic steroids (>10 mg/day prednisone equivalents), uncontrolled brain metastases, prior treatment with an anti-CTLA-4 agent or any known infection with HIV, Hepatitis B or C. The trial was approved by the local Institutional Review Board (IRB, Sourasky medical center Helsinky committee) and the Israeli Ministry of Health.

Methods. All patients eligible for participation in the compassionate use program signed a written informed consent. Patients were allocated to one single arm of Nivolumab $3 \mathrm{mg} / \mathrm{kg}$ administered intravenously every 2 weeks. Patients were staged by a whole-body CT scan or by PET-CT. Re-staging studies were carried out every four Nivolumab cycles. Patients with disease progression or symptomatic deterioration were excluded from the program, while those with controlled disease and clinical benefit continued treatment. The use of bisphosphonates or Denosumab was allowed in patisents with skeletal metastases. Data was assessed in order to find real-life response rate and time to progression.

Statistical analysis. We have used GraphPad Prism 4 software (GraphPad Software, Inc., La Jolla, CA, USA) for Survival and progression free survival analyses and curve preparation.

\section{Results}

Patient characteristics. A total of 77 patients (females, 34; males, 43) with age range of 33-99 years (median, 68 years) were enrolled into this cohort from June 2015 through December 2016. All patients had documented NSCLC: 13 (16.9\%) with squamous cell carcinoma and $64(83.1 \%)$ with non-squamous carcinoma (58 adenocarcinoma, 1 adeno-squamous, 1 large cell, and 4 not otherwise specified). Patients' characteristics are depicted in Table I. 44 (57.1\%) patients were non-smokers, $31(40.3 \%)$ current or former smokers and two (2.6\%) refused to supply smoking data. EGFR mutation analysis yielded mutations (mainly 19del and L858R) in 10/77 (13\%) of the patients. Metastatic involvement sites were lung in 64 patients, liver in 12 , adrenal in 10 , bone in 34 , and lymph nodes in 28 . Controlled brain metastases were documented in 11 patients. ECOG performance status was 1 in 52, 2 in 45 , and 3 in $3 \%$ of patients.

Treatment by nivolumab. A total number of 838 Nivolumab doses were administered (range: 1-41, median 7). Treatment was discontinued due to progressive disease (PD) in $46(59.7 \%)$ patients, toxicity in $5(6.5 \%)$, complete response (CR) in $1(1.3 \%)$, death in $15(19.5 \%)$, serious adverse events in $2(2.6 \%$ stroke in one, sepsis in one) and consent withdrawal in one (1.3\%). Seven patients remain on the drug. Nivolumab was given as the 1st line of treatment in $4 \%$ (elderly patients with good performance status, in whom chemotherapy was not an option), second line in $56 \%$, third line in $18 \%$, fourth in $17 \%$, and fifth in $5 \%$ of the patients.

Toxicity of nivolumab. Reported side effects were fatigue, rash, arthralgia, pruritus, diarrhea, nausea, loss of appetite, abdom-
Table I. Patient clinical characteristics $(n=77)$.

Characteristic

Total $(\%)$

Age

Median (range)

$68(33-99)$

Sex

Male

Female

Histology

Squamous

Non-squamous

Smoking status

Current smoking

Never or former smoking

Missing

Treatment line of nivolumab

First

Second

Third

Fourth

Fifth

ECOG performance status at cycle 1 0

1

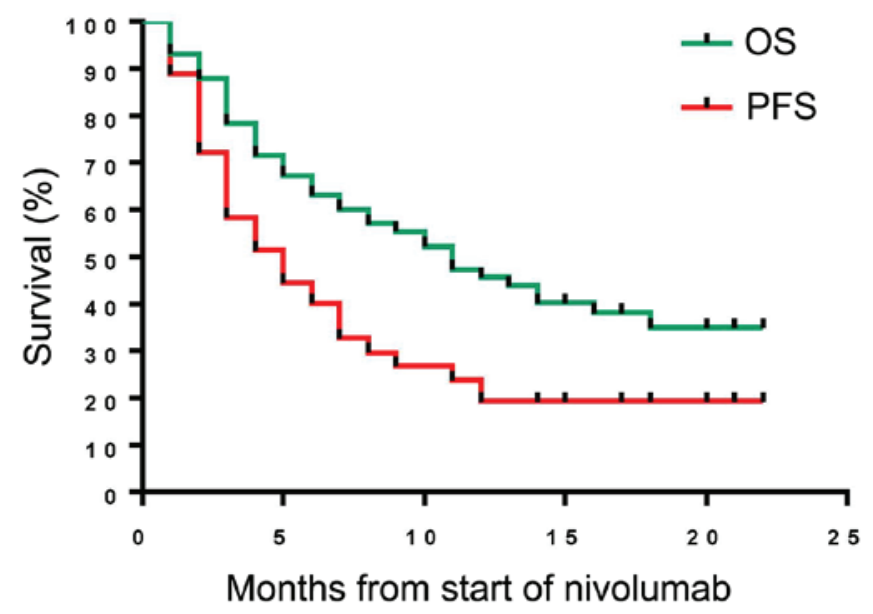

Figure 1. PFS and OS of patients treated with Nivolumab (programmed death-L1 unknown). PFS, progression free survival; OS, overall survival.

inal pain and pneumonitis. The most common was fatigue, documented in 52 patients. Side effects requiring steroids were diarrhea (10/15 patients) and pneumonitis (5/5 patients), only one pneumonitis patient had to stop treatment. Endocrinopathy, mostly hypothyroidism, was observed in 10 patients.

Response to nivolumab. Response assessment was performed in the 62 patients who got at least four cycles of Nivolumab. There was CR in 1 patient, partial response in 11, stable 
disease in 25 and PD in 25. The observed response rate was $19 \%$. The disease control rate was $60 \%$. Median progression free survival (mPFS) from the first dose to treatment interruption was 4 months. The PFS curve is depicted in Fig. 1. Survival data was analyzed after 22 months of follow-up. The overall survival was $34.9 \%$, while PFS was $19.3 \%$. Median overall survival from the first dose given and until patient's death or last follow up is 8 months. The OS curve is shown in Fig. 1. PFS and OS were similar for elderly vs. young patients (cut-off age 65), female vs. male sex, ECOG 1 vs. higher and early (1-2) vs. late line of treatment. All failures of Nivolumab occurred within the first 12 months of administration.

\section{Discussion}

Our results reflect real-life data on non-selected, PDL1 status unknown patients, including $48 \%$ patients with ECOG PS of 2 and 3 , and including $41 \%$ heavily pretreated patients. Yet, the results are similar to and even better than those seen in the already published RCT's, with ORR of $19 \%$ and median 22 -months-survival of $34.9 \%$. We could not isolate any characteristics that can predict who will benefit from immunotherapy. Side effects were almost always mild, and required discontinuation of treatment in less than $4 \%$ of patients.

Nivolumab was superior to Docetaxel in OS as well as side-effects profile, regardless of PDL1 status, in two RCT's. Checkmate 017 (7) was conducted on 272 patients with squamous cell histology, and showed mOS to be 9.2 months with Nivolumab, compared to 6 months with Docetaxel (HR, 0.59). Checkmate 057 (5) was performed on 582 patients with non-squamous histology, and again demonstrated superior OS of Nivolumab vs. Docetaxel (12.2 vs. 9.4 months; HR, 0.73).

Another PD1 inhibitor antibody, Pembrolizumab, was also superior to Docetaxel in a phase III trial. Keynote-010 (8) randomized 1,034 patients with NSCLC (all histology types) and at least $1 \%$ PDL1 expression, into either Pembrolizumab or Docetaxel treatment. The antibody resulted in significantly better OS (10.4 months and 12.7 months for both Pembrolizumab groups vs. 8.5 months, HR 0.71 and 0.61 respectively). Among patients with pdl1 expression above $50 \%$ OS reached an unprecedented 17.3 months.

PDL1 antibodies showed superiority over Docetaxel as well. Atezolizumab was tested in OAK trial (9), which randomized 1,225 patients with NSCLC (all histology types). OS was prolonged regardless of PDL1 expression (13.8 vs. 9.6 months, HR 0.73 ), while patients with $\geq 1 \%$ expression reached $\mathrm{mOS}$ of 15.7 months.

All immune checkpoint inhibitors were found to be less toxic than chemotherapy. All exhibited ORR of $14-20 \%$ with a median duration response of 16-20 months. Evidence is beginning to emerge regarding efficacy after longer follow-up. Gettinger et al described median 2-year-OS of $24 \%$ and median 3-year-OS of $18 \%$ in Nivolumab phase I expansion cohort (10). Brahmer et al presented as yet unpublished results of phase I Nivolumab in NSCLC, with an impressive median 5-year-OS of 16\% (11). Unpublished results were also presented of 2-year-OS of 29 and 23\% from Checkmate 057 and Checkmate 017, respectively (12).

In conclusion, Nivolumab is active and well tolerated in patients with NSCLC. This is based on highly selected population accrued for clinical trials and on non-selected cohorts reflecting daily service in thoracic oncology units. Our real-life data supports the results achieved in clinical trials, and supply a solid ground for continuing the use of Nivolumab in chemotherapy-pretreated patients with advanced disease.

\section{Acknowledgements}

Not applicable.

\section{Funding}

No funding was received.

\section{Availability of data and materials}

All data generated or analyzed during this study are included in this published article.

\section{Authors' contributions}

SS and OM performed the data analysis and wrote the paper.

\section{Ethics approval and consent to participate}

Not applicable.

\section{Patient consent for publication}

Not applicable.

\section{Competing interests}

The authors declare that they have no competing interests.

\section{References}

1. Shepherd FA, Dancey J, Ramlau R, Mattson K, Gralla R, O'Rourke M, Levitan N, Gressot L, Vincent M, Burkes R, et al: Prospective randomized trial of docetaxel versus best supportive care in patients with non-small-cell lung cancer previously treated with platinum-based chemotherapy. J Clin Oncol 18: 2095-2103, 2000.

2. Hanna N, Shepherd FA, Fossella FV, Pereira JR, De Marinis F, von Pawel J, Gatzemeier U, Tsao TC, Pless M, Muller T, et al: Randomized phase III trial of pemetrexed versus docetaxel in patients with non-small-cell lung cancer previously treated with chemotherapy. J Clin Oncol 22: 1589-1597, 2004.

3. Kim ES, Hirsh V, Mok T, Socinski MA, Gervais R, Wu YL, Li LY, Watkins CL, Sellers MV, Lowe ES, et al: Gefitinib versus docetaxel in previously treated non-small-cell lung cancer (INTEREST): A randomised phase III trial. Lancet 372: 1809-1818, 2008

4. Shepherd FA, Rodrigues Pereira J, Ciuleanu T, Tan EH, Hirsh V, Thongprasert S, Campos D, Maoleekoonpiroj S, Smylie M, Martins R, et al: Erlotinib in previously treated non-small-cell lung cancer. N Engl J Med 353: 123-132, 2005.

5. Borghaei H, Paz-Ares L, Horn L, Spigel DR, Steins M, Ready NE, Chow LQ, Vokes EE, Felip E, Holgado E, et al: Nivolumab versus docetaxel in advanced nonsquamous non-small-cell lung cancer. N Engl J Med 373: 1627-1639, 2015.

6. Kazandjian D, Suzman DL, Blumenthal G, Mushti S, He K, Libeg M, Keegan P and Pazdur R: FDA approval summary: Nivolumab for the treatment of metastatic non-small cell lung cancer with progression on or after platinum-based chemotherapy. Oncologist 21: 634-642, 2016. 
7. Brahmer J, Reckamp KL, Baas P, Crinò L, Eberhardt WE, Poddubskaya E, Antonia S, Pluzanski A, Vokes EE, Holgado E, et al: Nivolumab versus docetaxel in advanced squamous-cell non-small-cell lung cancer. N Engl J Med 373: $123-135,2015$.

8. Herbst RS, Baas P, Kim DW, Felip E, Pérez-Gracia JL, Han JY, Molina J, Kim JH, Arvis CD, Ahn MJ, et al: Pembrolizumab versus docetaxel for previously treated, PD-L1-positive, advanced non-small-cell lung cancer (KEYNOTE-010): A randomised controlled trial. Lancet 387: 1540-1550, 2016.

9. Rittmeyer A, Barlesi F, Waterkamp D, Park K, Ciardiello F, von Pawel J, Gadgeel SM, Hida T, Kowalski DM, Dols MC, et al: Atezolizumab versus docetaxel in patients with previously treated non-small-cell lung cancer (OAK): A phase 3, open-label, multicentre randomised controlled trial. Lancet 389: 255-265, 2017.

10. Gettinger SN, Horn L, Gandhi L, SpigelDR, Antonia SJ, RizviNA Powderly JD, Heist RS, Carvajal RD, Jackman DM, et al: Overall survival and long-term safety of nivolumab (Anti-Programmed Death 1 Antibody, BMS-936558, ONO-4538) in patients with previously treated advanced non-small-cell lung cancer. J Clin Oncol 33: 2004-2012, 2015.
11. Brahmer JR, Horn L, Jackman D, Spigel D, Antonia S, Hellmann M, Powderly J, Heist R, Sequist L, Smith DC, et al: Abstract CT077: Five-year follow-up from the CA209-003 study of nivolumab in previously treated advanced non-small cell lung cancer: Clinical characteristics of long-term survivors. Washington, DC. AACR Annual Meeting 77, April 1-5, 2017.

12. Borghaei H, Brahmer JR, Horn L, Ready N, Steins M, Felip E, Paz-Ares LG, Arrieta O, Barlesi F, Antonia SJ, et al: Nivolumab (nivo) vs docetaxel (doc) in patients (pts) with advanced NSCLC: CheckMate 017/057 2-y update and exploratory cytokine profile analyses. J Clin Oncol 34: 9025, 2016. 